\title{
Vacuna anti-influenza en niños: Estado del arte
}

\author{
Luis E. Vega-Briceño, Katia Abarca V. e lgnacio Sánchez D.
}

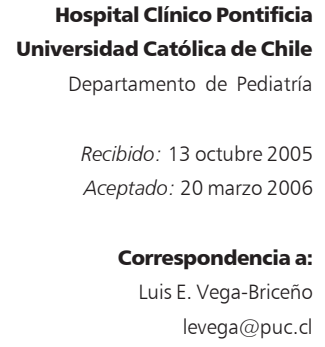

西

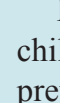
Influenza is an acute respiratory illness that year to year causes considerable morbidity and mortality:. All preventing influenza-like illness, laboratory-confirmed infection, and hospitalizations and deaths, demonstrating cost-effectiveness. Inactivated and live-attenuated vaccines have a similar efficacy profile-. Due to high influenza associated hospitalization rates in children, some countries have recommended incorporating influenza vaccination into the universal immunization program for healthy infants between 6-24 months of age. Considering that this public-health policy has been incorporated in Chile, local studies assessing its impact are needed.

Key words: influenza, vaccine, recommendations, children.

Palabras claves: influenza, vacunación, recomendaciones, niños.

\section{Introducción}

$\mathrm{E}$ 1 virus influenza es responsable anualmente de un elevado número de infecciones respiratorias en el mundo y en todos los grupos de edad ${ }^{1}$. Las particulares características de este virus, entre ellas su enorme capacidad de variación antigénica, hace que a pesar de los grandes esfuerzos y progresos en prevención y manejo, estas infecciones sigan produciendo un enorme impacto en términos de morbimortalidad $^{2}$.

Durante décadas, los principales grupos de riesgo identificados han sido los adultos mayores y los sujetos con afecciones crónicas como cardiopatías, asma bronquial, afecciones metabólicas (diabetes mellitus, insuficiencia renal crónica, entre otras) o inmunodeficiencias, quienes experimentan mayor frecuencia de hospitalizaciones, cuadros clínicos graves y una elevada tasa de mortalidad asociada a la infección ${ }^{3,4}$. Durante los últimos años y gracias a los avances en técnicas diagnósticas de infección viral se ha demostrado que los niños, especialmente los lactantes, constituyen un nuevo grupo de riesgo.

Por otra parte, la prevención de esta infección ha sido una materia en constante evolución, tanto por el desarrollo de nuevas vacunas como por el mejor conocimiento de su eficacia y seguridad. Recientemente se han introducido nuevas recomendaciones de inmunización en la edad pediátrica, motivándonos este escenario a revisar las evidencias e inconsistencias publicadas en la literatura, en torno a esta "dinámica" vacuna anti-influenza, con el especial interés de actualizar el conocimiento clínico y destacar los aspectos que aún requieren mayor investigación.

\section{Epidemiología}

Las infecciones respiratorias causadas por virus influenza tienen en la mayoría de los países un perfil epidemiológico claramente estacional (otoño y principios de invierno), presentándose anualmente la "temporada de influenza" que se caracteriza por ser de magnitud muy variable y a veces impredecible. En países sub-tropicales no existe esta marcada estacionalidad, pudiendo circular el virus durante todo el año. Durante la última década, la cepa que más ha circulado ha sido el tipo A con antígenos H3N2. Debido a las variaciones que experimenta el virus influenza $\mathrm{A}$ y a la presencia concomitante del virus influenza $\mathrm{B}$, es necesario que cada año se deba elaborar una nueva formulación polivalente de esta vacuna.

Los niños, comparativamente con adultos, experimentan una mayor frecuencia de infección por virus influenza ${ }^{2,5-8}$. La tasa de infección anual en ellos suele ser 1,5 a 3 veces mayor que la tasa registrada en adultos $^{2,5}$ : en promedio, 20 a $45 \%$ de los niños escolares y pre-escolares se enferman anualmente ${ }^{6,7}$ a diferencia de sólo 10 a $20 \%$ de la población adulta ${ }^{3}$. La tasa anual promedio de consulta médica por influenza se estima en 6 a $29 \%$ de la población, motivando hasta $30 \%$ de consumo innecesario de antimicrobianos ${ }^{8}$. Como se ha demostrado, los niños sanos bajo 2 años de edad presentan una elevada tasa de hospitalización 
(entre 190 y 400/100.000) y de complicaciones respiratorias, superando incluso las tasas de hospitalización de los adultos sobre 65 años ${ }^{6,8,9}$. Por lo frecuente que resulta la infección, así como por la elevada y prolongada excreción viral que ocurre en niños, han sido considerados los principales "vectores" responsables de la diseminación de este virus en la comunidad ${ }^{10}$.

El número de muertes atribuidas a este virus varía considerablemente según las diferentes regiones geográficas y temporadas; según el CDC de Atlanta, E.U.A., se estima que anualmente fallecen 50.000 personas a causa de este virus sólo en ese país ${ }^{3}$. Afortunadamente, la tasa de mortalidad infantil por influenza $(0,2-0,8 / 100.000)$ es significativamente inferior a la observada en adultos ${ }^{11}$. La variación en la gravedad que puede experimentar la infección en distintas temporadas queda graficada en la experiencia del hemisferio norte durante la temporada 2003-2004, cuando la epidemia se caracterizó por un inicio precoz y una mayor tasa de hospitalización por complicaciones respiratorias respecto a años previos 9 . En E.U.A., ese año murieron 143 niños; $41 \%$ de ellos eran niños bajo 2 años de edad y casi la mitad carecía de patologías crónicas de base ${ }^{4,9}$. Durante el mismo periodo se reportó un mayor número de neumonías asociadas a Streptococcus pneumoniae y Staphylococcus aureus ${ }^{12}$. También en nuestro país se observó un brote de inicio más precoz y de mayor gravedad en la temporada $2004^{12-14}$.

Habitualmente en Chile, la circulación del virus influenza se inicia alrededor de las semanas epidemiológicas 18-22, con variaciones anuales, tanto en su inicio como en su incidencia (Figura 1). En los últimos 3 años las tasas de notificación han fluctuado entre 20/ 100.000 el año 2003, 90/100.000 el año 2004 y 60/100.000 el año $2005^{14}$. Varias publicaciones nacionales concuerdan en demostrar el impacto de la influenza como causa de hospitalización en lactantes sanos ${ }^{13,15-18}$. La mayoría de las hospitalizaciones pediátricas ocurren en niños bajo 2 años de edad y alrededor de $60 \%$ en lactantes bajo 12 meses, afectando en su gran mayoría $(60-75 \%)$ a niños sanos, los que no son incluidos en los grupos de riesgo "tradicionales" con indicación de vacuna $^{15-18}$. Más de $50 \%$ presenta complicaciones como sobreinfección bacteriana pulmonar, otitis media aguda (OMA) o insuficiencia respiratoria ${ }^{15}$. Destaca que sólo entre 1 y $7 \%$ de los niños hospitalizados había recibido la vacuna anti-influenza antes del inicio de esa temporada ${ }^{13,15}$.

\section{Prevención}

La principal medida de prevención de la influenza la constituye la vacunación anual. Tradicionalmente la vacunación se ha indicado a los grupos de alto riesgo (adultos sobre 65 años de edad, personal de salud, pacientes con enfermedades cardio-respiratorias y metabólicas crónicas, consumo crónico de salicilatos y pacientes institucionalizados). En países como Canadá $^{19}$, Japón ${ }^{20}$ y Australia ${ }^{21}$, la recomendación de vacunación está dirigida preferentemente a estos grupos tradicionales de mayor riesgo. En la medida que se dispone de mayor información sobre identificación de nuevos grupos de riesgo, eficacia y seguridad de la vacuna $^{21,22}$, algunos países han ido ampliando sus recomendaciones de vacunación. Es así como E.U.A. ha incorporado en sus recomendaciones a mujeres embarazadas, adultos sobre 50 años de edad y más recientemente, a todos los niños entre 6 y 24 meses $^{9}$. En Europa, en general aún no se recomienda la inmunización a los niños sanos, aunque esta conducta ha sido materia de investigación reciente ${ }^{23}$.

En Chile el año 1982 se implementó el Programa Nacional de Vacunación Anti-influenza, dirigida a los grupos tradicionales de alto riesgo, llegando a ser uno de los países con mejores tasas de cobertura antiinfluenza en América Latina: 115 vacunados/1.000 habitantes en el año $2000^{23}$. El año 2004 se incorporó la vacunación de las mujeres embarazadas y el 2005 (medida que se hará efectiva a partir del 2006) a los lactantes sanos entre 6 y 24 meses (Tabla 1$)^{24}$.

\section{Vacunas disponibles}

Las vacunas anti-influenza disponibles actualmente en el mundo contienen virus inactivado (de uso parenteral), virus vivo atenuado (de uso nasal) o virosomas (de uso parenteral). Las vacunas inactivadas son elaboradas a partir de fragmentos de virus (split) o antígenos purificados (vacunas de sub-unidades). La eficacia, inmunogenicidad y seguridad de diferentes

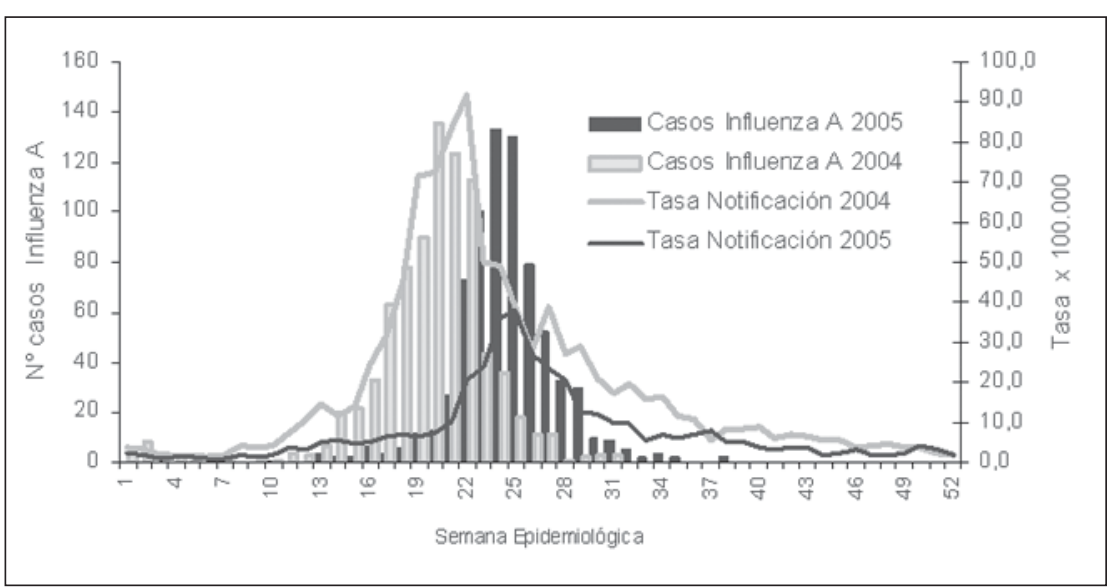

Figura 1. Tasa de notificación de influenza en centros centinelas, Chile 2003-2005. Fuente: MINSAL, ISP. Ref 14. 


Tabla 1. Recomendaciones actuales para el uso de
vacuna anti-influenza en Chile, MINSAL
1. Adultos sobre 65 años de edad
2. Pacientes con enfermedades crónicas: enfermedad pul-
monar obstructiva crónica, asma bronquial, fibrosis
quística, tuberculosis, neumoconiosis, enfisema pulmonar,
displasia broncopulmonar, diabetes mellitus insulina-de-
pendiente, diabetes mellitus no insulina-dependiente hos-
pitalizada el año previo, enfermedad coronaria isquémica,
enfermedad cardíaca crónica, insuficiencia renal crónica
en diálisis y discrasias sanguíneas severas
3. Pacientes portadores de inmunodeficiencias o con inmu-
nosupresión
4. Personal de salud o cuidadores de hogares de ancianos
5. Mujeres embarazadas con edad gestacional igual o ma-
yor a 14 semanas
6. Lactantes entre 6 meses y 2 años de edad
Tomado de: http://www.minsal.cl/ici/pandemiainfluenza/
anexo\%205.pdf

vacunas inactivadas es similar ${ }^{22}$. La vacuna que contiene virus vivo atenuado de uso nasal es elaborada con una cepa adaptada al frío, con capacidad de replicación a $25{ }^{\circ} \mathrm{C}$ (aparato respiratorio superior) e incapacidad de hacerlo en las mayores temperaturas del tracto respiratorio bajo. Se cree que podría estimular una mayor respuesta sistémica antigénica, mediante la generación de IgA local y linfocitos T citotóxicos. Esta vacuna ha sido recientemente licenciada por la FDA para su empleo en E.U.A. en personas entre $5 \mathrm{y}$ 49 años ${ }^{9}$. Las vacunas virosomales son desarrolladas a partir de vesículas pequeñas de $150 \mathrm{~nm}$ que exponen espículas de la hemaglutinina del virus. La unión de ellas con las células presentadoras de antígenos genera una fagocitosis, imitando una infección natural.

En nuestro medio, se encuentran disponibles vacunas inactivadas y virosomales, ambas de uso parenteral (vía subcutánea). El esquema de vacunación en niños depende de su edad y contactos previos con los antígenos del virus (Tabla 2). La vacuna no es recomendada en lactantes bajo seis meses de edad y está contraindicada en niños con antecedentes de anafilaxia demostrada al huevo, al timerosal o a dosis previas de la vacuna.

\section{Eficacia}

El análisis de la eficacia de las vacunas anti-influenza es bastante complejo, debiendo considerarse para medirla diversos factores como el diseño de los estudios, principalmente en lo que se refiere al parámetro usado para medir eficacia (capacidad para prevenir enfermedad clínica, enfermedad comprobada viroló-

\begin{tabular}{|c|c|c|}
\hline Edad & Dosis & $\begin{array}{c}\mathbf{N}^{\circ} \text { dosis en la } \\
\text { primera vacunación }\end{array}$ \\
\hline 6 meses a 3 años & $0,25 \mathrm{~mL}$ & $2 *$ \\
\hline 3 a 8 años & $0,5 \mathrm{~mL}$ & 2 * \\
\hline Sobre 8 años & $0,5 \mathrm{~mL}$ & 1 \\
\hline \multicolumn{3}{|c|}{$\begin{array}{l}\text { * La primera vez que el niño bajo } 9 \text { años de edad recibe } \\
\text { vacuna, y si no existe antecedente de influenza previa docu- } \\
\text { mentada, deberá recibir dos dosis de vacuna espaciadas por } \\
\text { al menos } 4 \text { semanas. }\end{array}$} \\
\hline
\end{tabular}

gicamente, consultas médicas, hospitalizaciones, muertes, episodios de OMA, uso de antimicrobianos, ausentismo laboral o escolar, etc). La eficacia además varía según la edad del huésped, el tipo de vacuna y la concordancia entre la cepa circulante y la contenida en la vacuna. Por ejemplo, durante la temporada de influenza 2003-2004 en el hemisferio norte se observó una baja eficacia de la vacuna, en relación con la circulación de una cepa A/Fujian no contenida en la vacuna; sólo $12 \%$ de los aislados correspondieron a la cepa A/Panamá presente en la vacuna usada ese año $0^{9,12}$.

Probablemente la mayor evidencia clínica reportada en relación a la eficacia de la vacunación anti-influenza corresponde a la experiencia de Japón ${ }^{20}$. En ese país, durante 25 años y de manera continua se vacunó a casi $85 \%$ de todos los niños escolares, medida que permitió prevenir entre 37.000 y 49.000 muertes por año, preferentemente en gente anciana; esto equivale a evitar una muerte por 420 niños vacunados. Este programa del gobierno japonés fue descontinuado en el año 1994, por la falta de estudios controlados que determinaran la seguridad de esta medida de salud pública.

Si bien la vacuna a virus inactivado tiene elevada eficacia (70-90\%) en adultos bajo 65 años de edad sin afecciones crónicas de base ${ }^{9}$, su eficacia es muy variable en niños entre 6 meses y 15 años de edad: 31 a $91 \%$ para influenza A y $45 \%$ para influenza $\mathrm{B}^{25,26}$. A pesar de estar incluidos en las recomendaciones de vacunación de E.U.A. en los últimos años, son muy pocos los estudios que han evaluado la eficacia de esta vacuna en lactantes bajo 2 años de edad ${ }^{27-29}$. Los estudios que han comparado la eficacia de la vacuna inactivada con la viva atenuada han dado resultados inciertos. Jefferson y cols evaluaron 14 estudios randomizados y controlados, concluyendo que las vacunas que contienen virus vivo atenuado podrían ser más eficaces ( 79 vs 65\%) que las vacunas a virus inactivo en niños, a partir de los 2 años ${ }^{27}$. Un reciente meta-análisis que 
evaluó 13 ensayos randomizados, controlados con más de 80.000 niños sanos, concluyó que la vacuna anti-influenza redujo significativamente la tasa de infección confirmada por laboratorio, hasta en $75 \%$ en el grupo vacunado, sin existir claras diferencias entre los niños que recibieron vacunas con virus vivo atenuado o virus inactivado ${ }^{30}$. Otros autores han mostrado conclusiones similares ${ }^{31}$. Se ha sugerido que la protección brindada por la vacuna anti-influenza podría ser diferente según la edad del niño ${ }^{28}$, siendo menor en niños bajo 2 años de edad. Un estudio bien diseñado, realizado en Japón, evaluó a 175 niños bajo 24 meses de edad, a quienes administraron dos dosis de vacuna anti-influenza durante 3 temporadas de influenza, observándose una tasa de infección similar entre los vacunados y los no vacunados ${ }^{29}$.

\section{Tolerancia y seguridad}

Se han observado síntomas locales en 3 a $27 \%$ de los niños vacunados (dolor, eritema o edema en el sitio de inyección) y síntomas sistémicos en 4 a 16\% (principalmente fiebre y coriza); la frecuencia de estos síntomas no difiere de los reportados en los niños que reciben placebo ${ }^{32,33}$. Respecto a seguridad, no se observaron eventos adversos serios en más de 250.000 niños bajo 18 años vacunados ${ }^{34}$. En el grupo de niños bajo 24 meses de edad, se ha reportado con baja frecuencia fiebre, exantemas urticariales o no especificados y convulsiones, siendo la mayoría de éstas asociadas a fiebre ${ }^{35}$. Los autores de este estudio concluyen que la vacuna anti-influenza es bastante segura en lactantes, pero que debe tenerse precaución en los niños con antecedentes familiares o personales de convulsión febril.

\section{Costo-efectividad}

La alta tasa de morbilidad asociada a influenza sugiere que la inmunización universal es un modelo económicamente atractivo ${ }^{35}$. Para hacer un análisis de costos debe considerarse la necesidad de revacunación anual debido a los cambios antigénicos menores (drift) que experimenta el virus influenza y la necesidad de administrar dos dosis durante el primer año de inmunización en los niños hasta 9 años de edad. Ya que la gravedad de la temporada influenza varía cada año de manera impredecible (desde formas leves hasta mortales), el costo de una vacunación universal podría llegar a ser extremadamente alto cuando se considera la población sana durante los años en que la enfermedad se presenta en forma leve ${ }^{36}$. Dos estudios- uno de ellos empleando un modelo matemático- que evaluaron la relación costo-beneficio en niños entre 6 meses y 14 años (con y sin factores de riesgo), determinaron que la vacunación era sustancialmente ventajosa en redu- cir la enfermedad y los costos producidos, especialmente en los grupos de alto riesgo $0^{37,38}$. Aunque la probabilidad de muerte fue baja $\mathrm{a}^{37}$, representó la variable con el mayor efecto susceptible de modulación; sin embargo, se requieren altas tasas de cobertura y expandir esta experiencia a otras temporadas de influenza, antes de obtener conclusiones definitivas.

\section{Limitaciones en la cobertura}

A pesar de la existencia de Programas Nacionales de Inmunización y recomendaciones explícitas, la cobertura de vacunación en niños en la mayoría de los países está lejos de ser la deseada. Las coberturas de vacunación en niños con afecciones crónicas alcanzadas actualmente en E.U.A. son del orden de 27 a $48 \%{ }^{12,39}$. Existen posibles factores que dificultan esta óptima cobertura. Muchos padres e incluso algunos miembros del personal de salud piensan, erróneamente, que los síntomas asociados a la vacunación son peores que la misma enfermedad o que puede producir una "pequeña gripe". La vacuna en general produce una baja tasa de reacciones adversas; el producto inyectable en uso es inactivado y está compuesto de fracciones antigénicas del virus por lo que no tiene capacidad biológica de producir infección. Lo mismo ocurre con la vacuna virosomal.

Por otro lado, los padres deben conocer que la vacuna anti-influenza es específica para el virus influenza, por lo que no se puede esperar que proteja contra otros virus respiratorios que también circulan en forma estacional junto con el virus influenza. Debe reconocerse que la eficacia de esta vacuna varía según las características del virus circulante (la cual varía en forma anual) y de las condiciones inmunitarias del hospedo. Es recomendable que los padres y médicos estén informados que la vacuna es bien tolerada y segura en niños ${ }^{23,27}$. Poehling y cols comprobaron que el factor más importante para un buen cumplimiento de vacunación anti-influenza en niños es la recomendación que hace el pediatra: cuando éste recomienda la vacuna, $70 \%$ de los niños la recibe; mientras que cuando no la recomienda, sólo $3 \%$ la recibe ${ }^{40}$.

\section{Inconsistencias}

Existe información que demuestra inconsistencia en la eficacia de la vacunación anti-influenza en niños con afecciones respiratorias crónicas como asma bronquial $^{26,41,42}$. Un reciente estudio, randomizado, controlado y ciego que evaluó a 697 niños asmáticos entre 6 y 18 años de edad, durante dos temporadas de influenza, no demostró una disminución significativa en el número, gravedad o duración de las exacerbaciones bronquiales respecto al grupo no vacunado ${ }^{41}$. Debe considerarse que el estudio no determinó la eficacia de 
la vacuna en prevenir infección, sino sólo en evitar las exacerbaciones agudas de asma bronquial. Ya que sólo un reducido número de infecciones respiratorias produce crisis de asma bronquial, se requeriría un gran número de sujetos para lograr este propósito.

La OMA es una complicación frecuente de la infección por virus influenza en niños; ocurre en $20 \%$ o más de los niños bajo 6 años de edad ${ }^{43}$. Diversos estudios han demostrado una reducción de 30 a $44 \%$ en la incidencia de OMA en niños correctamente vacuna$\operatorname{dos}^{43-45}$. Sin embargo, estos estudios presentan serias limitaciones en su diseño: tamaño de muestra pequeño y no representativo, o falta de un diseño aleatorio y ciego, por lo que sus resultados no pueden ser extrapolados fácilmente. Hoberman y colaboradores evaluaron 786 niños entre 6 y 24 meses de edad, en un estudio randomizado $2: 1$, doble ciego, controlado durante dos temporadas consecutivas de influenza, con seguimiento clínico durante 12 meses, no encontrando diferencias en la incidencia de OMA o complicaciones relacionadas respecto al grupo placebo ${ }^{25}$. Por lo expuesto, se requieren más estudios para establecer conclusiones definitivas.

\section{Conclusiones}

Parece existir acuerdo en que la vacunación antiinfluenza debe ser recomendada a todos los niños con afecciones crónicas de base. Si bien su eficacia es variable, los argumentos para incluir lactantes sanos entre 6 y 24 meses de edad sugieren su empleo rutinario; considerando especialmente la buena tolerancia y seguridad observada en niños pequeños. Diversos estudios controlados han demostrado que la vacuna anti-influenza es capaz de evitar episodios graves, hospitalización y significativos costos económicos, al menos en aquellas temporadas en que la vacuna se asemeja bien con la cepa circulante. Es deseable que cada país evalúe sus propias políticas de vacunación considerando estudios de costo-beneficio, especialmente si vamos a recomendar una mayor cobertura de vacunación anti-influenza en niños. Si ésta va ha ser una medida de salud pública, se requieren estudios bien diseñados que evalúen su real impacto en Chile.

\section{Resumen}

La influenza es una enfermedad respiratoria aguda con elevada tasa de morbilidad y mortalidad anual. Todos los niños, tanto sanos como aquellos de alto riesgo son susceptibles a la infección. La vacuna antiinfluenza es eficaz en prevenir síntomas asociados a influenza, infección demostrada por laboratorio, hospitalizaciones y muertes y ha demostrado ser una medida costo-efectiva. No se han observado claras diferencias entre las vacunas inactivadas y las nuevas vacunas elaboradas con virus vivo atenuado. Por la elevada tasa de hospitalización que los afecta, algunos países han resuelto recomendar la vacunación universal de los lactantes sanos entre 6 y 24 meses de edad. Considerando que esta medida de salud pública también ha sido incorporada en Chile, serían necesarios estudios locales que evalúen su real impacto en nuestro medio.

\section{Referencias}

1.- Monto A S. Viral respiratory infections in the community: epidemiology, agents, and interventions. Am J Med 1995; 99: 24S-27S.

2.- Nicholson K G, Wood J M, Zambon M. Influenza. Lancet 2003; 362: 1733-45.

3.- Thompson W, Shay D, Weintraub E, Brammer L, Cox N, Anderson L J, et al. Mortality associated with influenza and respiratory syncytial virus in the United States. JAMA 2003; 289: 179-86.

4.- Bhat N, Wright J, Broder $\mathrm{K}$ et al. Influenzaassociated deaths among children in the United States, 2003-2004. N Engl J Med 2005; 353: 2559-67.

5.- Monto A S, Sullivan K M. Acute respiratory illness in the community. Frequency of illness and the agents involved. Epidemiol Infect 1993; 110: 145-60.

6.- Izurieta H S, Thompson W W, Kramarz P, Shay D K, Davis R L, DeStefano F, et al. Influenza and the rates of hospitalization for respiratory disease among infants and young children. N Engl J Med 2000; 342: 232-9.

7.- Neuzil K M, Wright P F, Mitchel E F Jr, Griffin MR. The burden of influenza illness in children with asthma and other chronic medical conditions. J Pediatr 2000; 137: 856-64.

8.- Neuzil K M, Mellen B G, Wright P F, Mitchel E F Jr, Griffin MR. The effect of influenza on hospitalizations, outpatient visits, and courses of antibiotics in children. N Engl J Med 2000; 342: 225-31.

9.- Harper S A, Fukuda K, Uyeki T M, Cox N J, Bridges C B; Centers for Disease Control and
Prevention (CDC). Advisory Committee on Immunization Practices (ACIP). Prevention and control of influenza: Recommendations of the Advisory Committee on Immunization Practices (ACIP). MMWR Morbid Mortal Wkly Rep 2004; 53 (RR-6): $1-40$

10.- Glezen W P. Emerging infections: pandemic influenza. Epidemiol Rev 1996; 18: 64-76.

11.- Reina J. New indications for the inactivated influenza vaccine in the pediatric population (2004-2005). An Pediatr (Barc) 2005; 63: 45-9.

12.- Centers for Disease Control and Prevention (CDC). Estimated influenza vaccination coverage among adults and children--United States, September 1, 2004-January 31, 2005. MMWR Morb Mortal Wkly Rep 2005; 54: 304-7. 
13.- Vega-Briceño L E, Potín M, Bertrand P, Sánchez I. Infección respiratoria por virus influenza en niños: ¿Qué aprendimos durante el año 2004? Rev Méd Chile 2005; 133 : 911-8.

14.- http://epi.minsal.cl/epi/html/bolets/reportes/ Influenza/influenza.htm. (accedido el 05 de marzo del 2006).

15.- Delpiano L, Guillén B, Casado M C. Comportamiento clínico-epidemiológico de la influenza en niños hospitalizados. Rev Chil Infect 2003; 20: 159-65.

16.- Lagos R, Avendaño L F, Levine M M. Systematic surveillance of influenza, syncytial respiratory, parainfluenza and adenovirus in children with acute respiratory infections. Rev Méd Chile 1999; 127: 106372 .

17.- Avendaño L F, Palomino M A, Larrañaga C. Surveillance for respiratory syncytial virus in infants hospitalized for acute lower respiratory infection in Chile (1989 to 2000). J Clin Microbiol 2003; 41: 4879-82.

18. - Avendaño L F, Céspedes A, Stecher X, Palomino M A. Influence of respiratory viruses, cold weather and air pollution in the lower respiratory tract infections in infants children. Rev Méd Chile 1999; 127: 1073-8.

19. - Health Canada. Statement of influenza vaccination for the 2002-2003 season. Can Commun Dis Rep 2002; 28: 1-20.

20.- Reichert T A, Sugaya N, Fedson D S, Glezen W P, Simonsen L, Tashiro M. The Japanese experience with vaccinating schoolchildren against influenza N Engl J Med 2001; 344: 889-96.

21.- Isaacs D. Should all Australian children be vaccinated against influenza? Med J Aust 2005; 182: 553-4.

22.- Principi N, Esposito S. Pediatric influenza prevention and control. Emerg Infect Dis 2004; 10: 574-80.

23.- van Essen G, Palache A, Forleo E, Fedson D. Influenza vaccination in 2000 : recommendations and vaccine use in 50 developed and rapidly developing countries. Vaccine 2003; 21: 1780-5.

24.- http://epi.minsal.cl/epi/html/enfer/ previnfluenza.htm (accedido el 05 de marzo del 2006.

25.- Hoberman A, Greenberg D P, Paradise J L, Rockette H E, Lave J R, Keamey D H, et al.
Effectiveness of inactivated influenza vaccine in preventing acute otitis media in young children: a randomized controlled trial. JAMA 2003; 290: 1608-16.

26.- Ruben F L. Inactivated influenza virus vaccines in children. Clin Infect Dis 2004; 38: 678-88.

27.- Jefferson T, Smith S, Demicheli V, Harnden A, Rivetti A, Di Pietrantonj C. Assessment of the efficacy and effectiveness of influenza vaccines in healthy children: systematic review. Lancet 2005; 365: 77380 .

28.- Zangwill K M, Belshe R B. Safety and efficacy of trivalent inactivated influenza vaccine in young children: a summary for the new era of routine vaccination. Pediatr Infect Dis J 2004; 23: 189-97.

29. - Maeda T, Shintani Y, Nakano K Terashima K, Yamada Y. Failure of inactivated influenza $\mathrm{A}$ vaccine to protect healthy children aged 6-24 months. Pediatr Int 2004; 46: 122-5.

30.- Negri E, Colombo C, Giordano L, Groth N, Apolone G, La Vecchia C. Influenza vaccine in healthy children: a meta-analysis. Vaccine 2005; 23: 2851-61.

31.- Beyer W, Palache A, de Jong J, Osterhaus A. Cold-adapted live influenza vaccine versus inactivated vaccine: systemic vaccine reactions, local and systemic antibody response, and vaccine efficacy. A metaanalysis. Vaccine 2002; 20: 1340-53.

32. - Neuzil K M, Edwards K M. Influenza vaccines in children. Semin Pediatr Infect Dis 2002 Jul; 13: 174-81.

33. - McMahon A W, Iskander J, Haber P, Chang S, Woo E J, Braun M M, et al. Adverse events after inactivated influenza vaccination among children less than 2 years of age: analysis of reports from the vaccine adverse event reporting system, 1990-2003. Pediatrics 2005; 115: 453-60.

34. - France E K, Glanz J M, Xu S, Davis R L, Black S B, Shinefield H R, et al. Safety of the trivalent inactivated influenza vaccine among children: a population-based study. Arch Pediatr Adolesc Med 2004; 158: 10316.

35.- Luce B R, Zangwill K M, Palmer C S, Mendelman P M, Yan L, Wolff M C, et al. Cost-effectiveness analysis of an intranasal influenza vaccine for the prevention of influenza in healthy children. Pediatrics 2001; 108: E24.

36.- Szilagyi P G, Iwane M K, Schaffer S, Humiston S G, Barth R, McInemy T, et al. Potential burden of universal influenza vaccination of young children on visits to primary care practices. Pediatrics 2003; 112: 821-8.

37.- Meltzer M I, Neuzil K M, Griffin M R, Fukuda K. An economic analysis of annual influenza vaccination of children. Vaccine 2005; 23: 1004-14.

38.- Esposito S, Marchisio P, Bosis S, Lambertini L, Claut L, Faelli N, et al. Clinical and economic impact of influenza vaccination on healthy children aged 2-5 years. Vaccine 2006; 24: 629-35.

39.- Langley J M, Faughnan M E. Prevention of influenza in the general population. Can Med Assoc J 2004; 171: 1213-22.

40.- Poehling K A, Speroff T, Dittus R S, Griffin M R, Hickson G B, Edwards K M. Predictors of influenza virus vaccination status in hospitalized children. Pediatrics 2001; 108: e99.

41.- Bueving H, Bernsen R, Jongste J, van Suijlekom-Smit LW, Rimmelzwaan G F, Osterhaus A D, et al. Influenza vaccination in children with asthma: randomized doubleblind placebo-controlled trial. Am J Respir Crit Care Med 2004; 169: 488-93.

42. - Kramarz P, Destefano F, Gargiullo P M, Chen R T, Lieu T A, Davis R L, et al. Does influenza vaccination prevent asthma exacerbations in children? J Pediatr 2001; 138: 306-10.

43.- Belshe R B, Mendelman P M, Treanor J, King J, Gruber W C, Piedra P, et al. The efficacy of live attenuated, cold-adapted, trivalent, intranasal influenza virus vaccine in children. N Engl J Med 1998; 338: 1405-12.

44.- Clements D A, Langdon L, Bland C, Walter E. Influenza A vaccine decreases the incidence of otitis media in 6 to 30-monthold children in day care. Arch Pediatr Adolesc Med 1995; 149: 1113-7.

45.- Marchisio P, Cavagna R, Maspes B, Gironi S, Esposito S, Lambertini L, et al. Efficacy of intranasal virosomal influenza vaccine in the prevention of recurrent acute otitis media in children. Clin Infect Dis 2002; 35: 168-74. 